\title{
A New Applied Approach for Executing Computations with Infinite and Infinitesimal Quantities
}

\author{
Yaroslav D. SERGEYEV \\ Dipartimento di Elettronica, Informatica e Sistemistica, Università della Calabria \\ 87030 Rende (CS), Italy \\ e-mail:yaro@si.deis.unical.it
}

Received: November 2007; accepted: February 2008

\begin{abstract}
A new computational methodology for executing calculations with infinite and infinitesimal quantities is described in this paper. It is based on the principle 'The part is less than the whole' introduced by Ancient Greeks and applied to all numbers (finite, infinite, and infinitesimal) and to all sets and processes (finite and infinite). It is shown that it becomes possible to write down finite, infinite, and infinitesimal numbers by a finite number of symbols as particular cases of a unique framework. The new methodology has allowed us to introduce the Infinity Computer working with such numbers (its simulator has already been realized). Examples dealing with divergent series, infinite sets, and limits are given.
\end{abstract}

Keywords: infinite and infinitesimal numbers, infinite unite of measure, numeral systems, infinite sets, divergent series.

\section{Introduction}

Throughout the whole history of humanity many brilliant thinkers studied problems related to the idea of infinity (Cantor, 1955; Cohen, 1966; Conway and Guy, 1996; Gödel, 1940; Hardy, 1910; Hilbert, 1902; Leibniz and Child, 2005; Newton, 1671; Robinson, 1996) and references given therein). To emphasize importance of the subject it is sufficient to mention that the Continuum Hypothesis related to infinity has been included by David Hilbert as the Problem Number One in his famous list of 23 unsolved mathematical problems (Hilbert, 1902) that have influenced strongly development of Mathematics in the XXth century.

There exist different ways to generalize traditional arithmetic for finite numbers to the case of infinite and infinitesimal numbers (Benci and Di Nasso, 2003; Cantor, 1955; Conway and Guy, 1996; Robinson, 1996) and references given therein). However, arithmetics developed for infinite numbers are quite different with respect to the finite arithmetic we are used to deal with. Moreover, very often they leave undetermined many operations where infinite numbers take part (for example, $\infty-\infty, \frac{\infty}{\infty}$, sum of infinitely many items, etc.) or use representation of infinite numbers based on infinite sequences of finite numbers. In spite of these crucial difficulties and due to enormous importance of the concept 
of infinity in science, people try to introduce infinity in their work with computers. We can mention the IEEE Standard for Binary Floating-Point Arithmetic containing representations for $+\infty$ and $-\infty$ and incorporation of these notions in the interval analysis implementations.

The point of view on infinity accepted nowadays takes its origins from the famous ideas of Georg Cantor (Cantor, 1955) who has shown that there exist infinite sets having different number of elements. However, it is well known that Cantor's approach leads to some situations that often are called by non mathematicians 'paradoxes'. The most famous and simple of them is, probably, Hilbert's paradox of the Grand Hotel. In a normal hotel having a finite number of rooms no more new guests can be accommodated if it is full. Hilbert's Grand Hotel has an infinite number of rooms (of course, the number of rooms is countable, because the rooms in the Hotel are numbered). Due to Cantor, if a new guest arrives at the Hotel where every room is occupied, it is, nevertheless, possible to find a room for him. To do so, it is necessary to move the guest occupying room 1 to room 2 , the guest occupying room 2 to room 3, etc. In such a way room 1 will be ready for the newcomer and, in spite of our assumption that there are no available rooms in the Hotel, we have found one.

This result is very difficult to be fully realized by anyone who is not a mathematician since in our every day experience in the world around us the part is always less than the whole and if a hotel is complete there are no places in it. In order to understand how it is possible to tackle the problem of infinity in such a way that Hilbert's Grand Hotel would be in accordance with the principle 'the part is less than the whole' let us consider a study published in Science by Peter Gordon (Gordon, 2004) where he describes a primitive tribe living in Amazonia - Pirahã - that uses a very simple numeral system ${ }^{1}$ for counting: one, two, many. For Pirahã, all quantities larger than two are just 'many' and such operations as $2+2$ and $2+1$ give the same result, i.e., 'many'. Using their weak numeral system Pirahã are not able to see, for instance, numbers 3, 4, 5, and 6, to execute arithmetical operations with them, and, in general, to say anything about these numbers because in their language there are neither words nor concepts for that. Moreover, the weakness of their numeral system leads to such results as

$$
\text { 'many' }+1=\text { 'many', 'many' }+2=\text { 'many', }
$$

which are very familiar to us in the context of views on infinity used in the traditional calculus

$$
\infty+1=\infty, \quad \infty+2=\infty .
$$

This observation leads us to the following idea: Probably our difficulty in working with infinity is not connected to the nature of infinity but is a result of inadequate numeral systems used to express numbers.

\footnotetext{
${ }^{1}$ We remind that numeral is a symbol or group of symbols that represents a number. The difference between numerals and numbers is the same as the difference between words and the things they refer to. A number is a concept that a numeral expresses. The same number can be represented by different numerals. For example, the symbols ' 3 ', 'three', and 'III' are different numerals, but they all represent the same number.
} 
In this paper, we describe a new methodology for treating infinite and infinitesimal quantities (examples of its usage see in (Sergeyev, 2003; Sergeyev, 2004a; Sergeyev, 2004b; Sergeyev, 2005; Sergeyev, 2006a; Sergeyev, 2006b; Sergeyev, 2007)) having a strong numerical character. Its description is given in Section 2. The new methodology allows us to introduce in Section 3 a new infinite unit of measure that is then used as the radix of a new positional numeral system. Section 4 shows that this system allows one to express finite, infinite, and infinitesimal numbers in a unique framework and to execute arithmetical operations with all of them. Section 5 discusses some applications of the new methodology. Section 6 establishes relations to some of the results of Georg Cantor. After all, Section 7 concludes the paper.

We close this Introduction by emphasizing that the goal of the paper is not to construct a complete theory of infinity and to discuss such concepts as, for example, 'set of all sets'. In contrast, the problem of infinity is considered from the point of view of applied Mathematics and theory and practice of computations - fields being among the main scientific interests (see, e.g., monographs (Sergeyev, 2003; Strongin and Sergeyev, 2000)) of the author. A new viewpoint on infinity is introduced in the paper in order to give possibilities to solve new and old (but with higher precision) applied problems. Educational issues (Mockus, 2006; Mockus, 2008; Sergeyev, 2006b) have also been taken in account. In this connection, it is worthy to notice that a new kind of computers - the Infinity Computer - able to execute computations with infinite and infinitesimal numbers introduced in this paper has been recently proposed and its software simulator has already been implemented (Sergeyev, 2004a; Sergeyev, 2004b; Sergeyev, 2006a).

\section{A New Computational Methodology}

The aim of this section is to introduce a new methodology that would allow one to work with infinite and infinitesimal quantities in the same way as one works with finite numbers. Evidently, it becomes necessary to define what does it mean in the same way. Usually, in modern Mathematics, when it is necessary to define a concept or an object, logicians try to introduce a number of axioms describing the object. However, this way is fraught with danger because of the following reasons. First of all, when we describe a mathematical object or concept we are limited by the expressive capacity of the language we use to make this description. A more rich language allows us to say more about the object and a weaker language - less (remind Pirahã that are not able to say a word about number 4). Thus, development of the mathematical (and not only mathematical) languages leads to a continuous necessity of a transcription and specification of axiomatic systems. Second, there is no any guarantee that the chosen axiomatic system defines 'sufficiently well' the required concept and a continuous comparison with practice is required in order to check the goodness of the accepted set of axioms. However, there cannot be again any guarantee that the new version will be the last and definitive one. Finally, the third limitation latent in axiomatic systems has been discovered by Gödel in his two famous incompleteness theorems (Gödel, 1931). 
In this paper, we introduce a different, significantly more applied and less ambitious view on axiomatic systems related only to utilitarian necessities to make calculations. We start by introducing three postulates that will fix our methodological positions with respect to infinite and infinitesimal quantities and Mathematics, in general. In contrast to the modern mathematical fashion that tries to make all axiomatic systems more and more precise (decreasing so degrees of freedom of the studied part of Mathematics), we just define a set of general rules describing how practical computations should be executed leaving so as much space as possible for further, dictated by practice, changes and developments of the introduced mathematical language. Speaking metaphorically, we prefer to make a hammer and to use it instead of describing what is a hammer and how it works.

Usually, when mathematicians deal with infinite objects (sets or processes) it is supposed (even by constructivists see, for example, (Markov Jr. and Nagorny, 1996)) that human beings are able to execute certain operations infinitely many times. For example, in a fixed numeral system it is possible to write down a numeral with any number of digits. However, this supposition is an abstraction (courageously declared by constructivists in (Markov Jr. and Nagorny, 1996)) because we live in a finite world and all human beings and/or computers finish operations they have started. In this paper, this abstraction is not used and the following postulate is adopted.

Postulate 1. We postulate existence of infinite and infinitesimal objects but accept that human beings and machines are able to execute only a finite number of operations.

Thus, we accept that we shall never be able to give a complete description of infinite processes and sets due to our finite capabilities. Particularly, this means that we accept that we are able to write down only a finite number of symbols to express numbers.

The second postulate that will be adopted is due to the following consideration. In natural sciences, researchers use tools to describe the object of their study and the used instruments influence results of observations. When physicists see a black dot in their microscope they cannot say: the object of observation is the black dot. They are obliged to say: the lens used in the microscope allows us to see the black dot and it is not possible to say anything more about the nature of the object of observation until we shall not change the instrument - the lens or the microscope itself - by a more precise one.

Due to Postulate 1, the same happens in Mathematics when studying natural phenomena, numbers, and objects that can be constructed by using numbers. Numeral systems used to express numbers are among the instruments of observations used by mathematicians. Usage of powerful numeral systems gives the possibility to obtain more precise results in mathematics in the same way as usage of a good microscope gives the possibility to obtain more precise results in Physics. However, the capabilities of the tools will be always limited due to Postulate 1. Thus, following natural sciences, we accept the second postulate.

Postulate 2. We shall not tell what are the mathematical objects we deal with; we just shall construct more powerful tools that will allow us to improve our capacities to observe and to describe properties of mathematical objects.

Particularly, this means that from this applied point of view, axiomatic systems do not define mathematical objects but just determine formal rules for operating with certain 
numerals reflecting some properties of the studied mathematical objects. For example, axioms for real numbers are considered together with a particular numeral system $\mathcal{S}$ used to write down numerals and are viewed as practical rules (associative and commutative properties of multiplication and addition, distributive property of multiplication over addition, etc.) describing operations with the numerals. The completeness property is interpreted as a possibility to extend $\mathcal{S}$ with additional symbols (e.g., $e, \pi, \sqrt{2}$, etc.) taking care of the fact that the results of computations with these symbols agree with the facts observed in practice. As a rule, the assertions regarding numbers that cannot be expressed in a numeral system are avoided (e.g., it is not supposed that real numbers form a field).

After all, we want to treat infinite and infinitesimal numbers in the same manner as we are used to deal with finite ones, i.e., by applying the philosophical principle of Ancient Greeks 'The part is less than the whole'. This principle, in our opinion, very well reflects organization of the world around us but is not incorporated in many traditional infinity theories where it is true only for finite numbers.

Postulate 3. We adopt the principle 'The part is less than the whole' to all numbers (finite, infinite, and infinitesimal) and to all sets and processes (finite and infinite).

Due to this declared applied statement, such concepts as bijection, numerable and continuum sets, cardinal and ordinal numbers cannot be used in this paper because they belong to theories working with different assumptions. However, the approach proposed here does not contradict Cantor. In contrast, it evolves his deep ideas regarding existence of different infinite numbers in a more applied way.

It is important to notice that the adopted Postulates impose also the style of exposition of results in the paper: we first introduce new mathematical instruments, then show how to use them in several areas of Mathematics, introducing each item as soon as it becomes indispensable for the problem under consideration.

Let us introduce now the main methodological idea of the paper by studying a situation arising in practice and related to the necessity to operate with extremely large quantities (see (Sergeyev, 2003) for a detailed discussion). Imagine that we are in a granary and the owner asks us to count how much grain he has inside it. Of course, nobody counts the grain seed by seed because the number of seeds is enormous.

To overcome this difficulty, people take sacks, fill them in with seeds, and count the number of sacks. It is important that nobody counts the number of seeds in a sack. If the granary is huge and it becomes difficult to count the sacks, then trucks or even big train waggons are used. Of course, we suppose that all sacks contain the same number of seeds, all trucks - the same number of sacks, and all waggons - the same number of trucks. At the end of the counting we obtain a result in the following form: the granary contains 14 waggons, 54 trucks, 18 sacks, and 47 seeds of grain. Note, that if we add, for example, one seed to the granary, we can count it and see that the granary has more grain. If we take out one waggon, we again are able to say how much grain has been subtracted.

Thus, in our example it is necessary to count large quantities. They are finite but it is impossible to count them directly by using an elementary unit of measure, $u_{0}$, (seeds in our example) because the quantities expressed in these units would be too large. Therefore, people are forced to behave as if the quantities were infinite. 
To solve the problem of 'infinite' quantities, new units of measure, $u_{1}, u_{2}$, and $u_{3}$, are introduced (units $u_{1}$-sacks, $u_{2}$ - trucks, and $u_{3}$ - waggons). The new units have the following important peculiarity: all the units $u_{i+1}$ contain a certain number $K_{i}$ of units $u_{i}$ but this number, $K_{i}$, is unknown. Naturally, it is supposed that $K_{i}$ is the same for all instances of the units $u_{i+1}$. Thus, numbers that it was impossible to express using only the initial unit of measure are perfectly expressible in the new units we have introduced in spite of the fact that the numbers $K_{i}$ are unknown.

This key idea of counting by introduction of new units of measure will be used in the paper to deal with infinite quantities together with the idea of separate count of units with different exponents used in traditional positional numeral systems.

\section{The Infinite Unit of Measure}

The infinite unit of measure is expressed by the numeral (1) called grossone and is introduced as the number of elements of the set, $\mathbb{N}$, of natural numbers. Remind that the usage of a numeral indicating totality of the elements we deal with is not new in Mathematics. It is sufficient to mention the theory of probability (axioms of Kolmogorov) where events can be defined in two ways. First, as union of elementary events; second, as a sample space, $\Omega$, of all possible elementary events (or its parts $\Omega / 2, \Omega / 3$, etc.) from which some elementary events have been excluded (or added in case of parts of $\Omega$ ). Naturally, the latter way to define events becomes particularly useful when the sample space consists of infinitely many elementary events.

Grossone is introduced by describing its properties (similarly, in order to pass from natural to integer numbers a new element - zero - is introduced by describing its properties) postulated by the Infinite Unit Axiom (IUA) consisting of three parts: Infinity, Identity, and Divisibility. This axiom is added to axioms for real numbers (remind that we consider axioms in sense of Postulate 2). Thus, it is postulated that associative and commutative properties of multiplication and addition, distributive property of multiplication over addition, existence of inverse elements with respect to addition and multiplication hold for grossone as for finite numbers ${ }^{2}$. Let us introduce the axiom and then give comments on it.

Infinity. Any finite natural number $n$ is less than grossone, i.e., $n<$ (1).

Identity. The following relations link (1) to identity elements 0 and 1

$$
0 \cdot(1)=(1) \cdot 0=0, \quad(1)-(1)=0, \quad \frac{(1)}{(1)}=1, \quad(1)^{0}=1, \quad 1^{\mathbb{1}}=1, \quad 0^{\mathbb{1}}=0
$$

\footnotetext{
${ }^{2}$ It is important to emphasize that we speak about axioms of real numbers in sense of Postulate 2, i.e., axioms define formal rules of operations with numerals in a given numeral system. Therefore, if we want to have a numeral system including grossone, we should fix also a numeral system to express finite numbers. In order to concentrate our attention on properties of grossone, this point will be investigated later.
} 
Divisibility. For any finite natural number $n$ sets $\mathbb{N}_{k, n}, 1 \leqslant k \leqslant n$, being the $n$th parts of the set, $\mathbb{N}$, of natural numbers have the same number of elements indicated by the numeral $\frac{1}{n}$ where

$$
\mathbb{N}_{k, n}=\{k, k+n, k+2 n, k+3 n, \ldots\}, \quad 1 \leqslant k \leqslant n, \quad \bigcup_{k=1}^{n} \mathbb{N}_{k, n}=\mathbb{N}
$$

The first part of the introduced axiom - Infinity - is quite clear. In fact, we want to describe an infinite number, thus, it should be larger than any finite number. The second part of the axiom - Identity - tells us that (1) behaves itself with identity elements 0 and 1 as all other numbers. In reality, we could even omit this part of the axiom because, due to Postulate 3, all numbers should be treated in the same way and, therefore, at the moment we have told that grossone is a number, we have fixed usual properties of numbers, i.e., the properties described in Identity, associative and commutative properties of multiplication and addition, distributive property of multiplication over addition, existence of inverse elements with respect to addition and multiplication. The third part of the axiom - Divisibility - is the most interesting, it is based on Postulate 3. Let us first illustrate it by an example.

EXAMPLE 3.1. If we take $n=1$, then $\mathbb{N}_{1,1}=\mathbb{N}$ and Divisibility tells that the set, $\mathbb{N}$, of natural numbers has (1) elements. If $n=2$, we have two sets $\mathbb{N}_{1,2}$ and $\mathbb{N}_{2,2}$

$$
\begin{aligned}
& \mathbb{N}_{1,2}=\{1, \quad 3, \quad 5, \quad 7, \ldots\} \\
& \mathbb{N}_{2,2}=\{2, \quad 4, \quad 6, \quad \ldots\}
\end{aligned}
$$

and they have $\frac{1}{2}$ elements each. If $n=3$, then we have three sets

$$
\begin{aligned}
\mathbb{N}_{1,3} & =\{1, \quad 4, \\
\mathbb{N}_{2,3} & =\left\{\begin{array}{rlr}
2, & 5, & \ldots
\end{array}\right\} \\
\mathbb{N}_{3,3} & =\left\{\begin{array}{lll}
3, & 6, & \ldots
\end{array}\right\}
\end{aligned}
$$

and they have $\frac{(1)}{3}$ elements each.

It is important to emphasize that to introduce $\frac{(1)}{n}$ we do not try to count elements $k, k+n, k+2 n, k+3 n, \ldots$ one by one in (2). In fact, we cannot do this due to Postulate 1. By using Postulate 3 , we construct the sets $\mathbb{N}_{k, n}, 1 \leqslant k \leqslant n$, by separating the whole, i.e., the set $\mathbb{N}$, in $n$ parts (this separation is highlighted visually in formulae (3) and (4)). Again due to Postulate 3, we affirm that the number of elements of the $n$th part of the set, i.e., $\frac{1}{n}$, is $n$ times less than the number of elements of the whole set, i.e., than (1). In terms of our granary example (1) can be interpreted as the number of seeds in the sack. Then, if the sack contains (1) seeds, its $n$th part contains $n$ times less quantity, i.e., $\frac{1}{n}$ seeds. Note 
that, since the numbers $\frac{1}{n}$ have been introduced as numbers of elements of sets $\mathbb{N}_{k, n}$, they are integer.

The new unit of measure allows us to calculate easily the number of elements of sets being union, intersection, difference, or product of other sets of the type $\mathbb{N}_{k, n}$. Due to our accepted methodology, we do it in the same way as these measurements are executed for finite sets. Let us consider two simple examples (a general rule for determining the number of elements of infinite sets having a more complex structure will be given in Section 5) showing how grossone can be used for this purpose.

EXAMPLE 3.2. Let us determine the number of elements of the set $A_{k, n}=\mathbb{N}_{k, n} \backslash\{a\}$, $a \in \mathbb{N}_{k, n}, n \geqslant 1$. Due to the IUA, the set $\mathbb{N}_{k, n}$ has $\frac{1}{n}$ elements. The set $A_{k, n}$ has been constructed by excluding one element from $N_{k, n}$. Thus, the set $A_{k, n}$ has $\frac{1}{n}-1$ elements. The granary interpretation can be also given for the number $\frac{1}{n}-1$ : the number of seeds in the $n$th part of the sack minus one seed. For $n=1$ we have (1) -1 interpreted as the number of seeds in the sack minus one seed.

EXAMPLE 3.3. Let us consider the following two sets

$$
\begin{aligned}
& B_{1}=\{4,9,14,19,24,29,34,39,44,49,54,59,64,69,74,79, \ldots\} \\
& B_{2}=\{3,14,25,36,47,58,69,80,91,102,113,124,135, \ldots\}
\end{aligned}
$$

and determine the number of elements in the set $B=\left(B_{1} \cap B_{2}\right) \cup\{3,4,5,69\}$. It follows immediately from the IUA that $B_{1}=\mathbb{N}_{4,5}, B_{2}=\mathbb{N}_{3,11}$. Their intersection

$$
B_{1} \cap B_{2}=\mathbb{N}_{4,5} \cap \mathbb{N}_{3,11}=\{14,69,124, \ldots\}=\mathbb{N}_{14,55}
$$

and, therefore, due to the IUA, it has $\frac{1}{55}$ elements. Finally, since 69 belongs to the set $\mathbb{N}_{14,55}$ and 3, 4, and 5 do not belong to it, the set $B$ has $\frac{1}{55}+3$ elements. The granary interpretation: this is the number of seeds in the 55 th part of the sack plus three seeds.

One of the important differences of the new approach with respect to the non-standard analysis consists of the fact that (1) $\in \mathbb{N}$ because grossone has been introduced as the quantity of natural numbers (similarly, the number 5 being the number of elements of the set $\{1,2,3,4,5\}$ is the largest element in this set). The new numeral (1) allows one to write down the set, $\mathbb{N}$, of natural numbers in the form

$$
\mathbb{N}=\{1,2,3, \quad \cdots \quad(1)-3, \text { (1) }-2, \text { (1) }-1, \text { (1) }\}
$$

where the numerals

$$
\text { ... (1) }-3 \text {, (1) }-2 \text {, (1) - 1, (1) }
$$

indicate infinite natural numbers. 
It is important to emphasize that in the new approach the set (5) is the same set of natural numbers

$$
\mathbb{N}=\{1,2,3, \ldots\}
$$

we are used to deal with and infinite numbers (6) also take part of $\mathbb{N}$. Both records, (5) and (7), are correct and do not contradict each other. They just use two different numeral systems to express $\mathbb{N}$. Traditional numeral systems do not allow us to see infinite natural numbers that we can observe now thanks to (1). Similarly, Pirahã are not able to see finite natural numbers greater than 2. In spite of this fact, these numbers (e.g., 3 and 4) belong to $\mathbb{N}$ and are visible if one uses a more powerful numeral system. Thus, we have the same object of observation - the set $\mathbb{N}$ - that can be observed by different instruments - numeral systems - with different accuracies (see Postulate 2).

Now the following obvious question arises: Which natural numbers can we express by using the new numeral (1)? Suppose that we have a numeral system, $\mathcal{S}$, for expressing finite natural numbers and it allows us to express $K_{\mathcal{S}}$ numbers (not necessary consecutive) belonging to a set $\mathcal{N}_{\mathcal{S}} \subset \mathbb{N}$. Note that due to Postulate $1, K_{\mathcal{S}}$ is finite. Then, addition of (1) to this numeral system will allow us to express also infinite natural numbers $\frac{i(1)}{n} \pm k \leqslant(1)$ where $1 \leqslant i \leqslant n, k \in \mathcal{N}_{\mathcal{S}}, n \in \mathcal{N}_{\mathcal{S}}$ (note that since $\frac{(1)}{n}$ are integers, $\frac{i(1)}{n}$ are integers too). Thus, the more powerful system $\mathcal{S}$ is used to express finite numbers, the more infinite numbers can be expressed but their quantity is always finite, again due to Postulate 1 . The new numeral system using grossone allows us to express more numbers than traditional numeral systems thanks to the introduced new numerals but, as it happens for all numeral systems, its abilities to express numbers are limited.

EXAMPLE 3.4. Let us consider the numeral system, $\mathcal{P}$, of Pirahã able to express only numbers 1 and 2 (the only difference will be in the usage of numerals ' 1 ' and ' 2 ' instead of original numerals $I$ and $I I$ used by Pirahã). If we add to $\mathcal{P}$ the new numeral (1), we obtain a new numeral system (we call it $\widehat{\mathcal{P}}$ ) allowing us to express only ten numbers represented by the following numerals

$$
\underbrace{1,2}_{\text {finite }}, \cdots \underbrace{\frac{(1)}{2}-2, \frac{1}{2}-1, \frac{1}{2}, \frac{1}{2}+1, \frac{1}{2}+2}_{\text {infinite }}, \cdots \underbrace{(1)-2,(1)-1,(1)}_{\text {infinite }} .
$$

The first two numbers in (8) are finite, the remaining eight are infinite, and dots show natural numbers that are not expressible in $\widehat{\mathcal{P}}$. As a consequence, $\widehat{\mathcal{P}}$ does not allow us to execute such operation as $2+2$ or to add 2 to $\frac{1}{2}+2$ because their results cannot be expressed in it. Of course, we do not say that results of these operations are equal (as Pirahã do for operations $2+2$ and $2+1$ ). We just say that the results are not expressible in $\widehat{\mathcal{P}}$ and it is necessary to take another, more powerful numeral system if we want to execute these operations.

Note that crucial limitations discussed in Example 3.4 hold for sets, too. As a consequence, the numeral system $\mathcal{P}$ allows us to define only the sets $\mathbb{N}_{1,2}$ and $\mathbb{N}_{2,2}$ among 
all possible sets of the form $\mathbb{N}_{k, n}$ from (2) because we have only two finite numerals, ' 1 ' and ' 2 ', in $\mathcal{P}$. This numeral system is too weak to define other sets of this type because numbers greater than 2 required for these definition are not expressible in $\mathcal{P}$. These limitations have a general character and are related to all questions requiring a numerical answer (i.e., an answer expressed only in numerals, without variables). In order to obtain such an answer, it is necessary to know at least one numeral system able to express numerals required to write down this answer.

We are ready now to formulate the following important result being a direct consequence of the accepted methodological postulates.

\section{Theorem 3.1. The set $\mathbb{N}$ is not a monoid under addition.}

Proof. Due to Postulate 3, the operation (1) +1 gives us as the result a number greater than (1). Thus, by definition of grossone, (1) +1 does not belong to $\mathbb{N}$ and, therefore, $\mathbb{N}$ is not closed under addition and is not a monoid.

This result also means that adding the IUA to the axioms of natural numbers defines the set of extended natural numbers indicated as $\widehat{\mathbb{N}}$ and including $\mathbb{N}$ as a proper subset

$$
\widehat{\mathbb{N}}=\left\{1,2, \ldots,(1)-1,(1),(1)+1, \ldots,(1)^{2}-1,(1)^{2},(1)^{2}+1, \ldots\right\} \text {. }
$$

The extended natural numbers greater than grossone are also linked to sets of numbers and can be interpreted in the terms of grain.

EXAMPLE 3.5. Let us determine the number of elements of the set

$$
C=\left\{\left(a_{1}, a_{2}, \ldots, a_{m}\right): a_{i} \in \mathbb{N}, 1 \leqslant i \leqslant m\right\}
$$

The elements of $C$ are $m$-tuples of natural numbers. It is known from combinatorial calculus that if we have $m$ positions and each of them can be filled in by one of $l$ symbols, the number of the obtained $m$-tuples is equal to $l^{m}$. In our case, since $\mathbb{N}$ has grossone elements, $l=(1)$. Thus, the set $C$ has (1) ${ }^{m}$ elements. The granary interpretation: if we accept that the numbers $K_{i}$ from page 572 are such that $K_{i}=(1), 1 \leqslant i \leqslant m-1$, then (1) ${ }^{2}$ can be viewed as the number of seeds in the truck, (1) ${ }^{3}$ as the number of seeds in the train waggon, etc.

The set, $\widehat{\mathbb{Z}}$, of extended integer numbers can be constructed from the set, $\mathbb{Z}$, of integer numbers by a complete analogy and inverse elements with respect to addition are introduced naturally. For example, 7(1) has its inverse with respect to addition equal to -7(1) .

It is important to notice that, due to Postulates 1 and 2 , the new system of counting cannot give answers to all questions regarding infinite sets. What can we say, for instance, about the number of elements of the sets $\widehat{\mathbb{N}}$ and $\widehat{\mathbb{Z}}$ ? The introduced numeral system based on (1) is too weak to give answers to these questions. It is necessary to introduce in a way a more powerful numeral system by defining new numerals (for instance, (2), (3), etc). 
We conclude this section by the following remark. The IUA introduces a new number - the quantity of elements in the set of natural numbers - expressed by the new numeral (1). However, other numerals and sets can be used to state the idea of the axiom. For example, the numeral 1 can be introduced as the number of elements of the set, $\mathbb{E}$, of even numbers and can be taken as the base of a numeral system. In this case, the IUA can be reformulated using the numeral $(1$ and numerals using it will be used to express infinite numbers. For example, the number of elements of the set, $\mathbb{O}$, of odd numbers will be expressed as $|\mathbb{O}|=|\mathbb{E}|=\boldsymbol{(}$ and $|\mathbb{N}|=2 \cdot \boldsymbol{1}$. We emphasize through this note that infinite numbers (similarly to the finite ones) can be expressed by various numerals and in different numeral systems.

\section{Arithmetical Operations in the New Numeral System}

We have already started to write down simple infinite numbers and to execute arithmetical operations with them without concentrating our attention upon this question. Let us consider it systematically.

\subsection{Positional Numeral System with Infinite Radix}

Different numeral systems have been developed to describe finite numbers. In positional numeral systems, fractional numbers are expressed by the record

$$
\left(a_{n} a_{n-1} \ldots a_{1} a_{0} \cdot a_{-1} a_{-2} \ldots a_{-(q-1)} a_{-q}\right)_{b},
$$

where numerals $a_{i},-q \leqslant i \leqslant n$, are called digits, belong to the alphabet $\{0,1, \ldots, b-1\}$, and the dot is used to separate the fractional part from the integer one. Thus, the numeral (10) is equal to the sum

$$
a_{n} b^{n}+a_{n-1} b^{n-1}+\cdots+a_{1} b^{1}+a_{0} b^{0}+a_{-1} b^{-1}+\cdots+a_{-(q-1)} b^{-(q-1)}+a_{-q} b^{-q} .
$$

Record (10) uses numerals consisting of one symbol each, i.e., digits $a_{i} \in\{0,1$, $\ldots, b-1\}$, to express how many finite units of the type $b^{i}$ belong to the number (11). Quantities of finite units $b^{i}$ are counted separately for each exponent $i$ and all symbols in the alphabet $\{0,1, \ldots, b-1\}$ express finite numbers.

To express infinite and infinitesimal numbers we shall use records that are similar to (10) and (11) but have some peculiarities. In order to construct a number $C$ in the new numeral positional system with base (1), we subdivide $C$ into groups corresponding to powers of (1):

$$
C=c_{p_{m}}(1)^{p_{m}}+\ldots+c_{p_{1}}(1)^{p_{1}}+c_{p_{0}}(1)^{p_{0}}+c_{p_{-1}}(1)^{p_{-1}}+\ldots+c_{p_{-k}}(1)^{p_{-k}}
$$

Then, the record

$$
C=c_{p_{m}}(1)^{p_{m}} \ldots c_{p_{1}}(1)^{p_{1}} c_{p_{0}}(1)^{p_{0}} c_{p_{-1}}(1)^{p_{-1}} \ldots c_{p_{-k}}(1)^{p_{-k}}
$$


represents the number $C$, where all numerals $c_{i} \neq 0$, they belong to a traditional numeral system and are called grossdigits. They express finite positive or negative numbers and show how many corresponding units (1 ${ }^{p_{i}}$ should be added or subtracted in order to form the number $C$. Grossdigits can be expressed by several symbols using positional systems, the form $\frac{Q}{q}$ where $Q$ and $q$ are integer numbers, or in any other finite numeral system.

Numbers $p_{i}$ in (13) called grosspowers can be finite, infinite, and infinitesimal (the introduction of infinitesimal numbers will be given soon), they are sorted in the decreasing order

$$
p_{m}>p_{m-1}>\ldots>p_{1}>p_{0}>p_{-1}>\ldots p_{-(k-1)}>p_{-k}
$$

with $p_{0}=0$.

In the traditional record (10), there exists a convention that a digit $a_{i}$ shows how many powers $b^{i}$ are present in the number and the radix $b$ is not written explicitly. In the record (13), we write (1) ${ }^{p_{i}}$ explicitly because in the new numeral positional system the number $i$ in general is not equal to the grosspower $p_{i}$. This gives possibility to write, for example, such a number as 7.6(1) ${ }^{244.5} 341^{32}$ having grospowers $p_{2}=244.5, p_{1}=$ 32 and grossdigits $c_{244.5}=7.6, c_{32}=34$ without indicating grossdigits equal to zero corresponding to grosspowers less than 244.5 and greater than 32 . Note also that if a grossdigit $c_{p_{i}}=1$ then we often write (1) ${ }^{p_{i}}$ instead of $11^{p_{i}}$.

Finite numbers in this new numeral system are represented by numerals having only one grosspower $p_{0}=0$. In fact, if we have a number $C$ such that $m=k=0$ in representation (13), then due to (1), we have $C=c_{0}()^{0}=c_{0}$. Thus, the number $C$ in this case does not contain grossone and is equal to the grossdigit $c_{0}$ being a conventional finite number expressed in a traditional finite numeral system.

Infinitesimal numbers are represented by numerals $C$ having only negative finite or infinite grosspowers. The following two numbers are examples of infinitesimals: $3(1)^{-3.2}$, 37(1) ${ }^{-2} 11(1)^{-15}$. The simplest infinitesimal number is ()$^{-1}=\frac{1}{1}$ being the inverse element with respect to multiplication for (1):

$$
\frac{1}{(1)} \cdot(1)=(1) \cdot \frac{1}{1}=1 \text {. }
$$

Note that all infinitesimals are not equal to zero. Particularly, $\frac{1}{1}>0$ because it is a result of division of two positive numbers. It also has a clear granary interpretation. Namely, if we have a sack containing (1) seeds, then one sack divided by the number of seeds in it is equal to one seed. Vice versa, one seed, i.e., $\frac{1}{1}$, multiplied by the number of seeds in the sack, (1), gives one sack of seeds. Note that the usage of infinitesimals as grosspowers can lead to more complex constructions, particularly, again to infinitesimals, see, e.g., the number (1) ${ }^{-1}(-1)(1)^{0}$.

Infinite numbers in this numeral system are expressed by numerals having at least one finite or infinite grosspower greater than zero. Thus, they have infinite parts and can also have a finite part and infinitesimal ones. If power (1) ${ }^{0}$ is the lowest in a number then we often write simply grossdigit $c_{0}$ without (1) ${ }^{0}$, for instance, we write $231^{14} 5$ instead of $23(1)^{14} 5(1)^{0}$. 
EXAMPLE 4.1. The left-hand expression below shows how to write down numbers in the new numeral system and the right-hand shows how the value of the number is calculated:

$$
\begin{aligned}
& 15(1)^{1.4(1)}(-17.2045)(1)^{3} 7(1)^{0} 52.1(1)^{-6} \\
& \quad=15(1)^{1.4(1)}-17.2045(1)^{3}+7(1)^{0}+52.11^{-6} .
\end{aligned}
$$

The number above has one infinite part having the infinite grosspower, one infinite part having the finite grosspower, a finite part, and an infinitesimal part.

Finally, numbers having a finite and infinitesimal parts can be also expressed in the new numeral system, for instance, the number $-3.51^{0}(-37)(1)^{-2} 11(1)^{-15(1+2.3}$ has a finite and two infinitesimal parts, the second of them has the infinite negative grosspower equal to $-15(1)+2.3$.

\subsection{Arithmetical Operations}

We start the description of arithmetical operations for the new positional numeral system by the operation of addition (subtraction is a direct consequence of addition and is thus omitted) of two given infinite numbers $A$ and $B$, where

$$
A=\sum_{i=1}^{K} a_{k_{i}}(1)^{k_{i}}, \quad B=\sum_{j=1}^{M} b_{m_{j}}\left(1^{m_{j}}, \quad C=\sum_{i=1}^{L} c_{l_{i}}\left(1^{l_{i}}\right.\right.
$$

and the result $C=A+B$ is constructed by including in it all items $a_{k_{i}}\left(1{ }^{k_{i}}\right.$ from $A$ such that $k_{i} \neq m_{j}, 1 \leqslant j \leqslant M$, and all items $b_{m_{j}}(1)^{m_{j}}$ from $B$ such that $m_{j} \neq k_{i}$, $1 \leqslant i \leqslant K$. If in $A$ and $B$ there are items such that $k_{i}=m_{j}$, for some $i$ and $j$, then this grosspower $k_{i}$ is included in $C$ with the grossdigit $b_{k_{i}}+a_{k_{i}}$, i.e., as $\left(b_{k_{i}}+a_{k_{i}}\right)(1)^{k_{i}}$.

EXAMPLE 4.2. We consider two infinite numbers $A$ and $B$, where

$$
A=16.5(1)^{44.2}(-12)(1)^{12} 17(1)^{0}, \quad B=6.23(1)^{3} 10.1(1)^{0} 15(1)^{-4.1} .
$$

Their sum $C$ is calculated as follows:

$$
\begin{aligned}
C & =A+B \\
& =16.5(1)^{44.2}+(-12)(1)^{12}+17(1)^{0}+6.23(1)^{3}+10.1(1)^{0}+15(1)^{-4.1} \\
& =16.5(1)^{44.2}-12(1)^{12}+6.23(1)^{3}+27.1(1)^{0}+15(1)^{-4.1} \\
& =16.5(1)^{44.2}(-12)(1)^{12} 6.23(1)^{3} 27.1(1)^{0} 15(1)^{-4.1} .
\end{aligned}
$$

The operation of multiplication of two numbers $A$ and $B$ in the form (15) returns, as the result, the infinite number $C$ constructed as follows:

$$
C=\sum_{j=1}^{M} C_{j}, \quad C_{j}=b_{m_{j}}(1)^{m_{j}} \cdot A=\sum_{i=1}^{K} a_{k_{i}} b_{m_{j}}(1)^{k_{i}+m_{j}}, \quad 1 \leqslant j \leqslant M .
$$


EXAMPLE 4.3. We consider two infinite numbers

$$
A=1()^{18}(-5)(1)^{2.4}(-3) 1^{1}, \quad B=-11^{1}{ }^{1} 0.71^{-3}
$$

and calculate the product $C=B \cdot A$. The first partial product $C_{1}$ is equal to

$$
\begin{aligned}
C_{1} & \left.=0.7(1)^{-3} \cdot A=0.7(1)^{-3}(1)^{18}-5(1)^{2.4}-3(1)^{1}\right) \\
& =0.7(1)^{15}-3.5\left(1^{-0.6}-2.11^{-2}=0.7(1)^{15}(-3.5)(1)^{-0.6}(-2.1)(1)^{-2} .\right.
\end{aligned}
$$

The second partial product, $C_{2}$, is computed analogously

$$
\left.C_{2}=-(1)^{1} \cdot A=-(1)^{1}\left({ }^{1}\right)^{18}-51^{2.4}-3(1)^{1}\right)=-(1)^{19} 5(1)^{3.4} 3(1)^{2} \text {. }
$$

Finally, the product $C$ is equal to

$$
C=C_{1}+C_{2}=-1(1)^{19} 0.7(1)^{15} 5(1)^{3.4} 3(1)^{2}(-3.5)(1)^{-0.6}(-2.1)(1)^{-2} \text {. }
$$

In the operation of division of a number $C$ by a number $B$ from (15), we obtain a result $A$ and a reminder $R$ (that can be also equal to zero), i.e., $C=A \cdot B+R$. The number $A$ is constructed as follows. The first grossdigit $a_{k_{K}}$ and the corresponding maximal exponent $k_{K}$ are established from the equalities

$$
a_{k_{K}}=c_{l_{L}} / b_{m_{M}}, \quad k_{K}=l_{L}-m_{M} .
$$

Then the first partial reminder $R_{1}$ is calculated as

$$
R_{1}=C-a_{k_{K}}(1)^{k_{K}} \cdot B
$$

If $R_{1} \neq 0$ then the number $C$ is substituted by $R_{1}$ and the process is repeated with a complete analogy. The grossdigit $a_{k_{K-i}}$, the corresponding grosspower $k_{K-i}$ and the partial reminder $R_{i+1}$ are computed by formulae (19) and (20) obtained from (17) and (18) as follows: $l_{L}$ and $c_{l_{L}}$ are substituted by the highest grosspower $n_{i}$ and the corresponding grossdigit $r_{n_{i}}$ of the partial reminder $R_{i}$ that, in turn, substitutes $C$ :

$$
\begin{gathered}
a_{k_{K-i}}=r_{n_{i}} / b_{m_{M}}, \quad k_{K-i}=n_{i}-m_{M}, \\
R_{i+1}=R_{i}-a_{k_{K-i}}(1)^{k_{K-i}} \cdot B, \quad i \geqslant 1 .
\end{gathered}
$$

The process stops when a partial reminder equal to zero is found (this means that the final reminder $R=0$ ) or when a required accuracy of the result is reached.

EXAMPLE 4.4. Let us divide the number $C=-101^{3} 16(1)^{0} 42(1)^{-3}$ by the number $B=5(1)^{3} 7$. For these numbers we have

$$
l_{L}=3, \quad m_{M}=3, \quad c_{l_{L}}=-10, \quad b_{m_{M}}=5 .
$$


It follows immediately from (17) that $a_{k_{K}}\left(1^{k_{K}}=-2(1)^{0}\right.$. The first partial reminder $R_{1}$ is calculated as

$$
\begin{aligned}
R_{1} & =-10(1)^{3} 16(1)^{0} 42(1)^{-3}-\left(-21^{0}\right) \cdot 5(1)^{3} 7 \\
& =-10(1)^{3} 16(1)^{0} 42(1)^{-3}+10(1)^{3} 14(1)^{0}=30(1)^{0} 42(1)^{-3}
\end{aligned}
$$

By a complete analogy we should construct $a_{k_{K-1}}$ (1) $^{k_{K-1}}$ by rewriting (17) for $R_{1}$. By doing so we obtain equalities

$$
30=a_{k_{K-1}} \cdot 5, \quad 0=k_{K-1}+3
$$

and, as the result, $a_{k_{K-1}}(1)^{k_{K-1}}=6(1)^{-3}$. The second partial reminder is

$$
R_{2}=R_{1}-6(1)^{-3} \cdot 5(1)^{3} 7=30(1)^{0} 42(1)^{-3}-301^{0} 42(1)^{-3}=0 .
$$

Thus, we can conclude that the reminder $R=R_{2}=0$ and the final result of division is $A=-2(1)^{0} 6(1)^{-3}$.

Let us now substitute the grossdigit 42 by 40 in $C$ and divide this new number $\widetilde{C}=-10(1)^{3} 16$ (1) ${ }^{0} 40(1)^{-3}$ by the same number $B=5(1)^{3} 7$. This operation gives us the same result $\widetilde{A}_{2}=A=-2(1)^{0} 6(1)^{-3}$ (where subscript 2 indicates that two partial reminders have been obtained) but with the reminder $\widetilde{R}=\widetilde{R}_{2}=-21^{-3}$. Thus, we obtain $\widetilde{C}=B \cdot \widetilde{A}_{2}+\widetilde{R}_{2}$. If we want to continue the procedure of division, we obtain $\widetilde{A}_{3}=-2(1)^{0} 6(1)^{-3}(-0.4)(1)^{-6}$ with the reminder $\widetilde{R}_{3}=0.28(1)^{-6}$. Naturally, it follows $\widetilde{C}=B \cdot \widetilde{A}_{3}+\widetilde{R}_{3}$. The process continues until a partial reminder $\widetilde{R}_{i}=0$ is found or when a required accuracy of the result will be reached.

\section{Examples of Problems where Computations with New Numerals Can Be Useful}

\subsection{The Work with Infinite Sequences}

We start by reminding traditional definitions of the infinite sequences and subsequences. An infinite sequence $\left\{a_{n}\right\}, a_{n} \in A, n \in \mathbb{N}$, is a function having as the domain the set of natural numbers, $\mathbb{N}$, and as the codomain a set $A$. A subsequence is a sequence from which some of its elements have been removed. The IUA allows us to prove the following result.

Theorem 5.1. The number of elements of any infinite sequence is less or equal to (1).

Proof. The IUA states that the set $\mathbb{N}$ has (1) elements. Thus, due to the sequence definition given above, any sequence having $\mathbb{N}$ as the domain has (1) elements.

The notion of subsequence is introduced as a sequence from which some of its elements have been removed. Thus, this definition gives infinite sequences having the number of members less than grossone. 
One of the immediate consequences of the understanding of this result is that any sequential process can have at maximum (1) elements. Due to Postulate 1, it depends on the chosen numeral system which numbers among (1) members of the process we can observe.

EXAMPLE 5.1. Let us consider the set, $\widehat{\mathbb{N}}$, of extended natural numbers from (9). Then, starting from the number 1 , the process of the sequential counting can arrive at maximum to (1)

$$
\underbrace{1,2,3,4, \ldots \text { (1) }-2,(1)-1,(1)}_{(1)},(1)+1,(1)+2,(1)+3, \ldots
$$

Starting from 3 it arrives at maximum to (1) +2

$$
1,2, \underbrace{3,4, \ldots \text { (1) }-2,(1)-1,(1),(1)+1,(1)+2}_{(1)},(1)+3, \ldots
$$

It becomes appropriate now to define the complete sequence as an infinite sequence containing (1) elements. For example, the sequence of natural numbers is complete, the sequences of even and odd natural numbers are not complete. Thus, the IUA imposes a more precise description of infinite sequences. To define a sequence $\left\{a_{n}\right\}$ it is not sufficient just to give a formula for $a_{n}$, we should determine (as it happens for sequences having a finite number of elements) the first and the last elements of the sequence. If the number of the first element is equal to one, we can use the record $\left\{a_{n}: k\right\}$ where $a_{n}$ is, as usual, the general element of the sequence and $k$ is the number (that can be finite or infinite) of members of the sequence.

EXAMPLE 5.2. Let us consider the following two sequences, $\left\{a_{n}\right\}$ and $\left\{c_{n}\right\}$ :

$$
\begin{aligned}
& \left\{a_{n}\right\}=\{5,10, \ldots 5(1-1), 5(1)\} \\
& \left\{b_{n}\right\}=\left\{5,10, \ldots 5\left(\frac{21}{5}-1\right), 5 \cdot \frac{21}{5}\right\} \\
& \left\{c_{n}\right\}=\left\{5,10, \ldots 5\left(\frac{41}{5}-1\right), 5 \cdot \frac{41}{5}\right\} .
\end{aligned}
$$

They have the same general element $a_{n}=b_{n}=c_{n}=5 n$ but they are different because they have different numbers of members. The first sequence has (1) elements and is thus complete, the other two sequences are not complete: $\left\{b_{n}\right\}$ has $\frac{2(1)}{5}$ elements and $\left\{c_{n}\right\}$ has $\frac{4(1)}{5}$ members.

In connection with this definition the following natural question arises inevitably. Suppose that we have two sequences, for example, $\left\{b_{n}: \frac{2(1)}{5}\right\}$ and $\left\{c_{n}: \frac{4(1)}{5}\right\}$ from (21) 
and (22). Can we create a new sequence, $\left\{d_{n}: k\right\}$, composed from both of them, for instance, as it is shown below

$$
b_{1}, b_{2}, \ldots b_{\frac{2 \mathbb{D}}{5}-2}, b_{\frac{2 \mathbb{D}}{5}-1}, b_{\frac{2 \mathbb{D}}{5}}, c_{1}, c_{2}, \ldots c_{\frac{4 \mathbb{D}}{5}-2}, c_{\frac{4(\mathbb{D}}{5}-1}, c_{\frac{4 \mathbb{D}}{5}}
$$

and which will be the value of the number of its elements $k$ ?

The answer is 'no' because due to the definition of the infinite sequence, a sequence can be at maximum complete, i.e., it cannot have more than (1) elements. Starting from the element $b_{1}$ we can arrive at maximum to the element $c_{\frac{3}{5}(1)}$ being the element number (1) in the sequence $\left\{d_{n}: k\right\}$ which we try to construct. Therefore, $k=(1)$ and

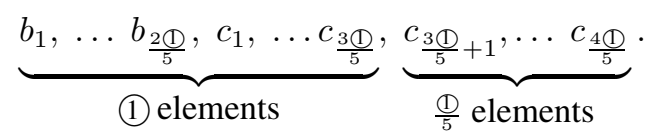

The remaining members of the sequence $\left\{c_{n}: \frac{4(1)}{5}\right\}$ will form the second sequence, $\left\{g_{n}: l\right\}$ having $l=\frac{4(1)}{5}-\frac{3(1)}{5}=\frac{1}{5}$ elements. Thus, we have formed two sequences, the first of them is complete and the second is not.

It is important to emphasize that the above consideration on the infinite sequences allows us to deal with recursively defined sets. Since such a set is constructed sequentially by a process, it can have at maximum (1) elements.

To conclude this subsection, let us return to Hilbert's paradox of the Grand Hotel presented in Section 2. In the paradox, the number of the rooms in the Hotel is countable. In our terminology this means that it has (1) rooms. When a new guest arrives, it is proposed to move the guest occupying room 1 to room 2, the guest occupying room 2 to room 3, etc. Under the IUA this procedure does not help because the guest from room (1) should be moved to room (1) +1 and the Hotel has only (1) rooms. Thus, when the Hotel is full, no more new guests can be accommodated - the result corresponding perfectly to Postulate 3 and the situation taking place in normal hotels with a finite number of rooms.

\subsection{Calculating Divergent Series}

Let us show how the new approach can be applied in such an important area as theory of divergent series. We consider two infinite series $S_{1}=10+10+10+\cdots$ and $S_{2}=$ $3+3+3+\cdots$. The traditional analysis gives us a very poor answer that both of them diverge to infinity. Such operations as, e.g., $\frac{S_{2}}{S_{1}}$ and $S_{2}-S_{1}$ are not defined.

Now, when we are able to express not only different finite numbers but also different infinite numbers, it is necessary to indicate explicitly the number of items in the sums $S_{1}$ and $S_{2}$ and it is not important if it is finite or infinite. To calculate the sum it is necessary that the number of items and the result are expressible in the numeral system used for calculations. It is important to notice that even though a sequence cannot have more than (1) elements, the number of items in a series can be greater than grossone because the process of summing up is not necessary executed by a sequential adding items. 
Let us suppose that the series $S_{1}$ has $k$ items and $S_{2}$ has $n$ items. We can then define sums (that can have a finite or an infinite number of items),

$$
S_{1}(k)=\underbrace{10+10+10+\cdots+10}_{k}, \quad S_{2}(n)=\underbrace{3+3+3+\cdots+3}_{n},
$$

calculate them, and execute arithmetical operations with the obtained results. The sums then are obviously calculated as $S_{1}(k)=10 k$ and $S_{2}(n)=3 n$. If, for instance, $k=n=$ 5 (1) then we obtain $S_{1}(5(1))=50(1), S_{2}(5$ (1) $)=15$ (1) and

$$
S_{2}(5(1)) / S_{1}(5(1))=0.3
$$

Analogously, if $k=3(1)$ and $n=10(1)$ we obtain $\left.S_{1}(3(1))=30(1), S_{2}(1)\right)=30$ (1) and it follows $\left.\left.S_{2}(1)\right)-S_{1}(31)\right)=0$.

If $k=3(1) 4$ (we remind that we use here a shorter way to write down this infinite number, the complete record is $3\left(1^{1} 41^{0}\right)$ and $n=10(1)$ we obtain $S_{1}\left(31{ }^{4}\right)=$ 30 (1) $\left.40, S_{2}(1)\right)=30$ (1) and it follows

$$
\begin{aligned}
& S_{1}(3(1) 4)-S_{2}(1)=30(1) 40-30(1)=40 . \\
& \left.S_{1}(3 \text { (1) } 2) / S_{2}(1)\right)=30 \text { (1) } 20 / 30(1)=11^{0} 0.666671^{-1}>0 .
\end{aligned}
$$

We conclude this subsection by studying the series $\sum_{i=1}^{\infty} \frac{1}{2^{i}}$. It is known that it converges to one. However, we are able to give a more precise answer. Due to Postulate 3 , the formula

$$
\sum_{i=1}^{k} \frac{1}{2^{i}}=1-\frac{1}{2^{k}}
$$

can be used directly for infinite $k$, too. For example, if $k=(1)$ then

$$
\sum_{i=1}^{(\mathbb{D}} \frac{1}{2^{i}}=1-\frac{1}{2^{\mathbb{D}}},
$$

where $\frac{1}{2^{\mathbb{1}}}$ is infinitesimal. Thus, the traditional answer $\sum_{i=1}^{\infty} \frac{1}{2^{i}}=1$ is a finite approximation to our more precise result using infinitesimals. More examples related to series can be found in (Sergeyev, 2007).

\subsection{Calculating Limits and Expressing Irrational Numbers}

Let us now discuss the problem of calculation of limits from the point of view of our approach. In traditional analysis, if a $\operatorname{limit}_{x \rightarrow a} f(x)$ exists, then it gives us a very poor - just one value - information about the behavior of $f(x)$ when $x$ tends to $a$. Now we can obtain significantly richer information because we are able to calculate $f(x)$ directly 
at any finite, infinite, or infinitesimal point that can be expressed by the new positional system even if the limit does not exist.

Thus, limits equal to infinity can be substituted by precise infinite numerals and limits equal to zero can be substituted by precise infinitesimal numerals ${ }^{3}$. This is very important for practical computations because these substitutions eliminate indeterminate forms.

EXAMPLE 5.3. Let us consider the following two limits

$$
\lim _{x \rightarrow+\infty}\left(5 x^{3}-x^{2}+10^{61}\right)=+\infty, \quad \lim _{x \rightarrow+\infty}\left(5 x^{3}-x^{2}\right)=+\infty .
$$

Both give us the same result, $+\infty$, and it is not possible to execute the operation

$$
\lim _{x \rightarrow+\infty}\left(5 x^{3}-x^{2}+10^{61}\right)-\lim _{x \rightarrow+\infty}\left(5 x^{3}-x^{2}\right) .
$$

that is an indeterminate form of the type $\infty-\infty$ in spite of the fact that for any finite $x$ it follows

$$
5 x^{3}-x^{2}+10^{61}-\left(5 x^{3}-x^{2}\right)=10^{61} .
$$

The new approach allows us to calculate exact values of both expressions, $5 x^{3}-x^{2}+10^{61}$ and $5 x^{3}-x^{2}+10$, at any infinite (and infinitesimal) $x$ expressible in the chosen numeral system. For instance, the choice $x=3(1)^{2}$ gives the value

$$
5\left(3(1)^{2}\right)^{3}-\left(3(1)^{2}\right)^{2}+10^{61}=135(1)^{6}-9(1)^{4} 10^{61}
$$

for the first expression and $135(1)^{6}-9(1)^{4}$ for the second one. We can easily calculate the difference of these two infinite numbers, thus obtaining the same result as we had for finite values of $x$ in (23):

$$
135(1)^{6}-9(1)^{4} 10^{61}-\left(135(1)^{6}-9(1)^{4}\right)=10^{61} .
$$

It is necessary to emphasize the fact that expressions can be calculated even when their limits do not exist. Thus, we obtain a very powerful tool for studying divergent processes.

EXAMPLE 5.4. The $\operatorname{limit}_{\lim _{n \rightarrow+\infty}} f(n), f(n)=(-1)^{n} n^{3}$, does not exist. However, we can easily calculate expression $(-1)^{n} n^{3}$ at different infinite points $n$. For instance, for $n=$ (1) it follows $f(1)=(1)^{3}$ because grossone is even and for the odd $n=0.5(1)-1$ it follows

$$
f(0.5(1)-1)=-(0.5(1)-1)^{3}=-0.125(1)^{3} 0.75(1)^{2}-1.5(1)^{1} 1 .
$$

\footnotetext{
${ }^{3}$ Naturally, if we speak about limits of sequences, $\lim _{n \rightarrow \infty} a(n)$, then $n \in \mathbb{N}$ and, as a consequence, it follows that $n$ should be less than or equal to grossone.
} 
Limits with the argument tending to zero can be considered analogously. In this case, we can calculate the corresponding expression at any infinitesimal point using the new positional system and obtain a significantly more reach information.

EXAMPLE 5.5. If $x$ is a fixed finite number then

$$
\lim _{h \rightarrow 0} \frac{(x+h)^{2}-x^{2}}{h}=2 x .
$$

In the new positional system we obtain

$$
\frac{(x+h)^{2}-x^{2}}{h}=2 x+h
$$

If, for instance, $h=(1)^{-1}$, the answer is $2 x(1)^{0}(1)^{-1}$, if $h=4.2(1)^{-2}$ we obtain the value $2 x(1)^{0} 4.2\left(1^{-2}\right.$, etc. Thus, the value of the limit (24), for a finite $x$, is just the finite approximation of the number (25) having finite and infinitesimal parts.

Let us make a remark regarding irrational numbers. Among their properties, they are characterized by the fact that we do not know any numeral system that would allow us to express them by a finite number of symbols used to express other numbers. Thus, special numerals $(e, \pi, \sqrt{2}, \sqrt{3}$, etc.) are introduced by describing their properties in a way (similarly, all other numerals, e.g., symbols ' 0 ' or ' 1 ', are introduced also by describing their properties). These special symbols are then used in analytical transformations together with ordinary numerals.

For example, it is possible to work directly with the symbol $e$ in analytical transformations by applying suitable rules defining this number together with numerals taking part in a chosen numeral system $\mathcal{S}$. At the end of transformations, the obtained result will be be expressed in numerals from $\mathcal{S}$ and, probably, in terms of $e$. If it is then required to execute some numerical computations, this means that it is necessary to substitute $e$ by a numeral (or numerals) from $\mathcal{S}$ that will allow us to approximate $e$ in some way.

The same situation takes place when one uses the new numeral system, i.e., while we work analytically we use just the symbol $e$ in our expressions and then, if we wish to work numerically we should pass to approximations. The new numeral system opens a new perspective on the problem of the expression of irrational numbers. Let us consider one of the possible ways to obtain an approximation of $e$, i.e., by using the limit

$$
e=\lim _{n \rightarrow+\infty}\left(1+\frac{1}{n}\right)^{n}=2.71828182845904 \ldots
$$

In our numeral system the expression $\left(1+\frac{1}{n}\right)^{n}$ can be written directly for finite and/or infinite values of $n$. For $n=(1)$ we obtain the number $e_{0}$ designated so in order to distinguish it from the record (26)

$$
e_{0}=\left(1+\frac{1}{(1)}\right)^{(1)}=\left(1^{0}\left(1^{-1}\right)^{\mathbb{1}}\right. \text {. }
$$


It becomes clear from this record why the number $e$ cannot be expressed in a positional numeral system with a finite base. Due to the definition of a sequence under the IUA, such a system can have at maximum (1) numerals - digits - to express fractional part of a number (see Subsection 5.5 for details) and, as it can be seen from (27), this quantity is not sufficient for $e$ because the item $\frac{1}{(1)^{(1)}}$ is present in it.

Naturally, it is also possible to construct more exotic $e$-type numbers by substituting (1) in (27) by any infinite number written in the new positional system with infinite base. For example, if we substitute (1) in (27) by (1) ${ }^{2}$ we obtain the number

$$
e_{1}=\left(1+\frac{1}{\mathbb{1}^{2}}\right)^{\mathbb{1}^{2}}=\left(\left(^{0}{ }^{0}()^{-2}\right)^{\mathbb{D}^{2}}\right.
$$

The numbers considered above take their origins in the limit (26). Similarly, other formulae leading to approximations of $e$ expressed in traditional numeral systems give us other new numbers that can be expressed in the new numeral system. The same way of reasoning can be used with respect to other irrational numbers, too.

\subsection{Measuring Infinite Sets with Elements Defined by Formulae}

We have already discussed in Section 3 how we calculate the number of elements for sets being results of the usual operations (intersection, union, etc.) with finite sets and infinite sets of the type $\mathbb{N}_{k, n}$. In order to have a possibility to work with infinite sets having a more general structure than the sets $\mathbb{N}_{k, n}$, we need to develop more powerful instruments. Suppose that we have an integer function $g(i)>0$ strictly increasing on indexes $i=1,2,3, \ldots$ and we wish to know how many elements are there in the set

$$
G=\{g(1), g(2), g(3), \ldots\}
$$

In our terminology this question has no any sense because of the following reason.

In the finite case, to define a set it is not sufficient to say that it is finite. It is necessary to indicate its number of elements explicitly as, e.g., in this example

$$
G_{1}=\{g(i): 1 \leqslant i \leqslant 5\}
$$

or implicitly, as it is made here:

$$
G_{2}=\{g(i): i \geqslant 1,0<f(i) \leqslant b\}
$$

where $b$ is finite.

Now we have mathematical tools to indicate the number of elements for infinite sets, too. Thus, analogously to the finite case and due to Postulate 3 , it is not sufficient to say that a set has infinitely many elements. It is necessary to indicate its number of elements explicitly or implicitly. For instance, the number of elements of the set

$$
G_{3}=\left\{g(i): 1 \leqslant i \leqslant(1)^{10}\right\}
$$


is indicated explicitly: the set $G_{3}$ has (1) ${ }^{10}$ elements.

If a set is given in the form (28) where $b$ is infinite, then its number of elements, $J$, can be determined as

$$
J=\max \{i: g(i) \leqslant b\}
$$

if we are able to determine the inverse function $g^{-1}(x)$ for $g(x)$. Then, $J=\left[g^{-1}(b)\right]$, where $[u]$ is integer part of $u$. Note that if $b=(1)$, then the set $G_{2} \subseteq \mathbb{N}$ since all its elements are integer, positive, and $g(i) \leqslant(1)$ due to (29).

EXAMPLE 5.6. Let us consider the following set, $A_{1}(k, n)$, having $g(i)=k+n(i-1)$,

$$
A_{1}(k, n)=\{g(i): i \geqslant 1, g(i) \leqslant(1)\}, \quad 1 \leqslant k \leqslant n, \quad n \in \mathbb{N} .
$$

It follows from the IUA that $A_{1}(k, n)=\mathbb{N}_{k, n}$ from (2). By applying (29) we find for $A_{1}(k, n)$ its number of elements

$$
J_{1}(k, n)=\left[\frac{1}{n}+k\right]=\left[\frac{1)-k}{n}\right]+1=\frac{(1)}{n}-1+1=\frac{(1)}{n} .
$$

EXAMPLE 5.7. Analogously, the set

$$
A_{2}(k, n, j)=\left\{k+n i^{j}: i \geqslant 0,0<k+n i^{j} \leqslant \text { (1) }\right\}, \quad 0 \leqslant k<n, n \in \mathbb{N}, j \in \mathbb{N},
$$

has $J_{2}(k, n, j)=\left[\sqrt[j]{\frac{1}{n}-k}\right]$ elements.

\subsection{Measuring Infinite Sets of Numerals and Their Comparison}

Let us calculate the number of elements in some well known infinite sets of numerals using the designation $|A|$ to indicate the number of elements of a set $A$.

Theorem 5.2. The number of elements of the set, $\mathbb{Z}$, of integers is $|\mathbb{Z}|=2(1) 1$.

Proof. The set $\mathbb{Z}$ contains (1) positive numbers, (1) negative numbers, and zero. Thus,

$$
|\mathbb{Z}|=(1)+(1)+1=211
$$

Traditionally, rational numbers are defined as ratio of two integer numbers. The new approach allows us to calculate the number of numerals in a fixed numeral system. Let us consider a numeral system $\mathbb{Q}_{1}$ containing numerals of the form

$$
\frac{p}{q}, \quad p \in \mathbb{Z}, \quad q \in \mathbb{Z}, \quad q \neq 0 .
$$

Theorem 5.3. The number of elements of the set, $\mathbb{Q}_{1}$, of rational numerals of the type (31) is $\left|\mathbb{Q}_{1}\right|=4 \mathbb{1}^{2} 2 \mathbb{1}^{1}$. 
Proof. It follows from Theorem 5.2 that the numerator of (31) can be filled in by 2(1) 1 and the denominator by 2 (1) numbers. Thus, number of all possible combinations is

$$
\left|\mathbb{Q}_{1}\right|=2(1) 1 \cdot 2(1)=4(1)^{2} 2(1)^{1} .
$$

It is necessary to notice that in Theorem 5.3 we have calculated different numerals and not different numbers. For example, in the numeral system $\mathbb{Q}_{1}$ the number 0 can be expressed by $2(1)$ different numerals

$$
\frac{0}{-(1)}, \frac{0}{-(1)+1}, \frac{0}{-(1)+2}, \ldots \frac{0}{-2}, \frac{0}{-1}, \frac{0}{1}, \frac{0}{2}, \ldots \frac{0}{(1)-2}, \frac{0}{(1)-1}, \frac{0}{1},
$$

and numerals such as $\frac{-1}{-2}$ and $\frac{1}{2}$ have been calculated as two different numerals. The following theorem determines the number of elements of the set $\mathbb{Q}_{2}$ containing numerals of the form

$$
-\frac{p}{q}, \frac{p}{q}, \quad p \in \mathbb{N}, \quad q \in \mathbb{N}
$$

and zero is represented by one symbol 0 .

Theorem 5.4. The number of elements of the set, $\mathbb{Q}_{2}$, of rational numerals of the type (32) is $\left|\mathbb{Q}_{2}\right|=2(1)^{2} 1$.

Proof. Let us consider positive rational numerals. The form of the rational numeral $\frac{p}{q}$, the fact that $p, q \in \mathbb{N}$, and the IUA impose that both $p$ and $q$ can assume values from 1 to (1). Thus, the number of all possible combinations is 1$)^{2}$. The same number of combinations we obtain for negative rational numbers and one is added because we count zero as well.

Let us now calculate the number of elements of the set, $\mathbb{R}_{b}$, of real numbers expressed by numerals in the positional system by the record

$$
\left(a_{n-1} a_{n-2} \ldots a_{1} a_{0} \cdot a_{-1} a_{-2} \ldots a_{-(q-1)} a_{-q}\right)_{b}
$$

where the symbol $b$ indicates the radix of the record and $n, q \in \mathbb{N}$.

Theorem 5.5. The number of elements of the set, $\mathbb{R}_{b}$, of numerals (33) is $\left|\mathbb{R}_{b}\right|=b^{2 \Phi}$.

Proof. In formula (33) defining the type of numerals we deal with there are two sequences of digits: the first one, $a_{n-1} a_{n-2} \ldots a_{1} a_{0}$, is used to express the integer part of the number and the second, $a_{-1} a_{-2} \ldots a_{-(q-1)} a_{-q}$, for its fractional part. Due to definition of sequence and the IUA, each of them can have at maximum (1) elements. Thus, it can be at maximum (1) positions on the left of the dot and, analogously, (1) positions on the right of the dot. Every position can be filled in by one of the $b$ digits from the alphabet $\{0,1, \ldots, b-1\}$. Thus, we have $b^{(\mathbb{D})}$ combinations to express the integer part of the number and the same quantity to express its fractional part. As a result, the positional numeral system using the numerals of the form (33) can express $b^{2 \perp}$ numbers. 
Note that the result of theorem 5.5 does not consider the practical situation of writing down concrete numerals. Obviously, the number of numerals of the type (33) that can be written in practice is finite and depends on the chosen numeral system for writing digits.

It is worthwhile to notice also that the traditional point of view on real numbers tells that there exist real numbers that can be represented in positional systems by two different infinite sequences of digits. In contrast, under the IUA all the numerals represent different numbers. In addition, minimal and maximal numbers expressible in $\mathbb{R}_{b}$ can be explicitly indicated.

EXAMPLE 5.8. For instance, in the decimal positional system $\mathbb{R}_{10}$ the numerals

$$
1 . \underbrace{999 \ldots 99}_{\text {(1) digits }}, \quad 2 . \underbrace{000 \ldots 00}_{(1) \text { digits }}
$$

represent different numbers and their difference is equal to

$$
2 . \underbrace{000 \ldots 00}_{\text {(1) digits }}-1 . \underbrace{999 \ldots 9}_{\text {(1) digits }}=0 . \underbrace{000 \ldots 01}_{\text {(1) digits }} .
$$

Analogously the smallest and the largest numbers expressible in $\mathbb{R}_{10}$ can be easily indicated. They are, respectively,

$$
-\underbrace{999 \ldots 9}_{\text {(1) digits }} \cdot \underbrace{999 \ldots 9}_{\text {(1) digits }}, \quad \underbrace{999 \ldots 9}_{\text {(1) digits }} \cdot \underbrace{999 \ldots 9}_{\text {(1) digits }} .
$$

Theorem 5.6. The sets $\mathbb{Z}, \mathbb{Q}_{1}, \mathbb{Q}_{2}$, and $\mathbb{R}_{b}$ are not monoids under addition.

Proof. The proof is obvious and is so omitted.

\section{Relations to Results of Georg Cantor}

It is obligatory to say in this occasion that the results presented above should be considered as a more precise analysis of the situation discovered by the genius of Cantor. He has proved, by using his famous diagonal argument, that the number of elements of the set $\mathbb{N}$ is less than the number of real numbers at the interval $[0,1)$ without calculating the latter. To do this he expressed real numbers in a positional numeral system. We have shown that this number will be different depending on the radix $b$ used in the positional system to express real numbers. However, all of the obtained numbers, $b^{2 \Phi}$, are more than the number of elements of the set of natural numbers, (1), and, therefore, the diagonal argument maintains its force.

We can now calculate the number of points of the interval $[0,1)$, of a line, and of the $N$-dimensional space. To do this we need a definition of the term point and mathematical tools to indicate a point. Since this concept is one of the most fundamental, it is very 
difficult to find an adequate definition. If we accept (as is usually done in modern Mathematics) that the point $x$ in an $N$-dimensional space is determined by $N$ numerals called coordinates of the point

$$
\left(x_{1}, x_{2}, \ldots x_{N-1}, x_{N}\right) \in \mathbb{S}^{N},
$$

where $\mathbb{S}^{N}$ is a set of numerals, then we can indicate the point $x$ by its coordinates and we are able to execute the required calculations. It is worthwhile to emphasize that we have not postulated that $\left(x_{1}, x_{2}, \ldots x_{N-1}, x_{N}\right)$ belongs to the $N$-dimensional set, $\mathbb{R}^{N}$, of real numbers as it is usually done because we can express coordinates only by numerals and, as we have shown above, different choices of numeral systems lead to various sets of numerals.

We should decide now which numerals we shall use to express coordinates of the points. Different variants can be chosen depending on the precision level we want to obtain. For example, if the numbers $0 \leqslant x<1$ are expressed in the form $\frac{p-1}{1}, p \in \mathbb{N}$, then the smallest positive number we can distinguish is $\frac{1}{(1)}$. Therefore, the interval $[0,1)$ contains the following (1) points

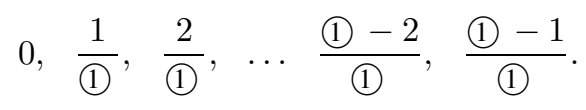

Then, due to the IUA and the definition of sequence, there are (1) intervals of the form $[a-1, a), a \in \mathbb{N}$, on the ray $x \geqslant 0$. Hence, this ray contains $(1)^{2}$ points and the whole line consists of $2{ }^{2}{ }^{2}$ points.

If we need a higher precision, within each interval

$$
\left[a-1+\frac{i-1}{(1)}, a-1+\frac{i}{1}\right), \quad a, i \in \mathbb{N}
$$

we can distinguish again (1) points and the number of points within each interval $[a-$ $1, a), a \in \mathbb{N}$, will become equal to $(1)^{2}$. Consequently, the number of the points on the line will be equal to $21^{3}$.

This situation is a direct consequence of Postulate 2 and is typical for natural sciences where it is well known that instruments influence the results of observations. It is similar as to work with a microscope: we decide the level of the precision we need and obtain a result which is dependent on the chosen level of accuracy. If we need a more precise or a more rough answer, we change the lens of our microscope.

Continuing the analogy with the microscope, we can also decide to change our microscope with a new one. In our terms this means to change the numeral system with another one. For instance, instead of the numerals considered above, we choose a positional numeral system to calculate the number of points within the interval $[0,1)$; then, as we have already seen before, we are able to distinguish $b^{\mathbb{E}}$ points of the form

$$
\left(. a_{-1} a_{-2} \ldots a_{-(1)-1)} a_{-(1)}\right)_{b}
$$


on it. Since the line contains 2(1) unit intervals, the whole number of points of this type on the line is equal to $2(1) b^{(\mathbb{D}}$.

In this example of counting, we have changed the tool to calculate the number of points within each interval, but used the old way to calculate the number of intervals, i.e., by natural numbers. If we are not interested in subdividing the line at intervals and want to obtain the number of the points on the line directly by using positional numerals of the type (33) with possible infinite $n$ and $q$, then we are able to distinguish at maximum $b^{21}$ points on the line.

Let us now return to the problem of comparison of infinite sets and consider Cantor's famous result showing that the number of points over the interval $(0,1)$ is equal to the number of points over the whole real line, i.e.,

$$
|\mathbb{R}|=|(0,1)|
$$

The proof of this counterintuitive fact is given by establishing a one-to-one correspondence between the elements of the two sets. Such a mapping can be done by using for example the function

$$
y=\tan (0.5 \pi(2 x-1)), \quad x \in(0,1)
$$

illustrated in Fig. 1. Cantor shows by using Fig. 1 that to any point $x \in(0,1)$ a point $y \in$ $(-\infty, \infty)$ can be associated and vice versa. Thus, he concludes that the requested oneto-one correspondence between the sets $\mathbb{R}$ and $(0,1)$ has been established and, therefore, this proves (34).

Our point of view is different: the number of elements is an intrinsic characteristic of each set (for both finite and infinite cases) that does not depend on any object outside the set. Thus, in Cantor's example from Fig. 1 we have (see Fig. 2) three mathematical

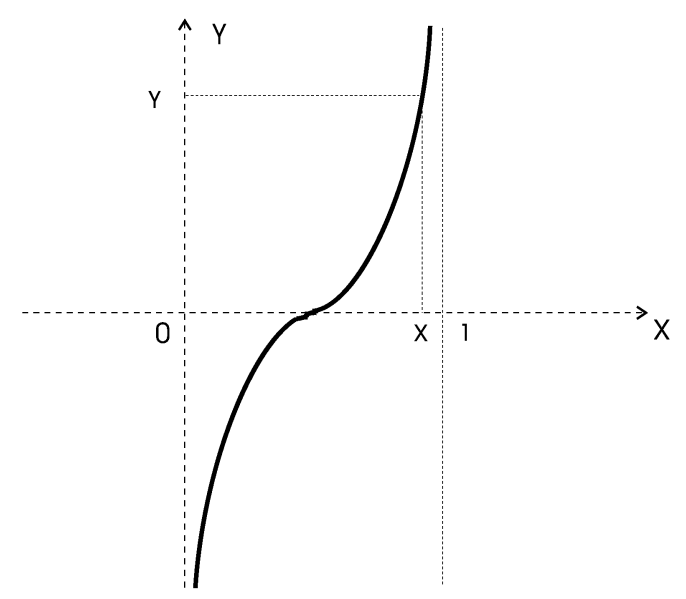

Fig. 1. Due to Cantor, the interval $(0,1)$ and the entire real number line have the same number of points. 


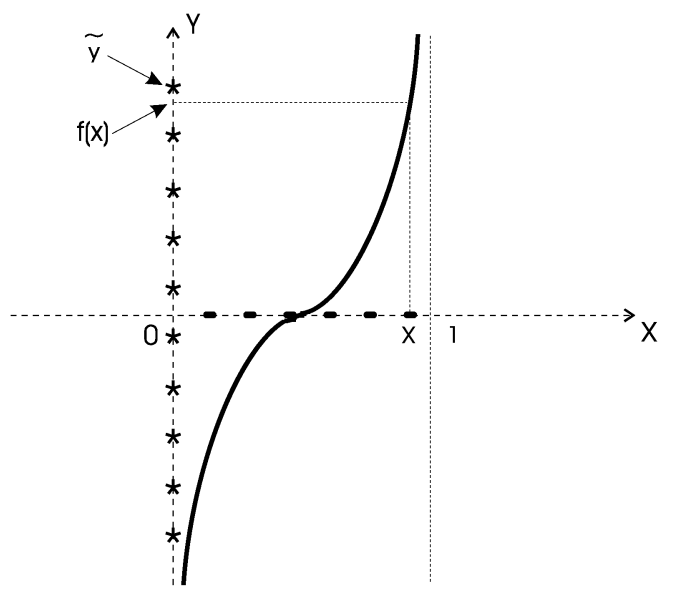

Fig. 2. Three independent mathematical objects: the set $X_{\mathcal{S}_{1}}$ represented by dots, the set $Y_{\mathcal{S}_{2}}$ represented by stars, and function (35).

objects: (i) a set, $X_{\mathcal{S}_{1}}$, of points over the interval $(0,1)$ which we are able to distinguish using a numeral system $\mathcal{S}_{1}$; (ii) a set, $Y_{\mathcal{S}_{2}}$, of points over the vertical real line which we are able to distinguish using a numeral system $\mathcal{S}_{2}$; (iii) the function (35) described using a numeral system $\mathcal{S}_{3}$. All these three mathematical objects are independent each other. The sets $X_{\mathcal{S}_{1}}$ and $Y_{\mathcal{S}_{2}}$ can have the same or different number of elements.

Thus, we are not able to evaluate $f(x)$ at any point $x$. We are able to do this only at points from $X_{\mathcal{S}_{1}}$. Of course, in order to be able to execute these evaluations it is necessary to conciliate the numeral systems $\mathcal{S}_{1}, \mathcal{S}_{2}$, and $\mathcal{S}_{3}$. The fact that we have made evaluations of $f(x)$ and have obtained the corresponding values does not influence minimally the numbers of elements of the sets $X_{\mathcal{S}_{1}}$ and $Y_{\mathcal{S}_{2}}$. Moreover, it can happen that the number $y=f(x)$ cannot be expressed in the numeral system $\mathcal{S}_{2}$ and it is necessary to approximate it by a number $\widetilde{y} \in \mathcal{S}_{2}$. This situation, very well known to computer scientists, is represented in Fig. 2.

Let us remind one more famous example related to the one-to-one correspondence and taking its origins in studies of Galileo Galilei: even numbers can be put in a one-toone correspondence with all natural numbers in spite of the fact that they are a part of them:

$$
\begin{array}{ll}
\text { even numbers: } & 2,4,6,8,10,12, \ldots \\
& \uparrow \uparrow \uparrow \uparrow \uparrow \uparrow \\
\text { natural numbers: } & 1,2,3,45, \quad 6, \ldots
\end{array}
$$

Again, our view on this situation is different since we cannot establish a one-to-one correspondence between the sets because they are infinite and we, due to Postulate 1, are able to execute only a finite number of operations. We cannot use the one-to-one correspondence as an executable operation when it is necessary to work with infinite sets. 
However, we already know that the number of elements of the set of natural numbers is equal to (1) and (1) is even. Since the number of elements of the set of even numbers is equal to $\frac{1}{2}$, we can write down not only initial (as it is usually done traditionally) but also the final part of (36)

$$
\begin{aligned}
& 2,4,6,8,10,12, \ldots \text { (1) }-4 \text {, (1) }-2 \text {, (1) } \\
& \uparrow \uparrow \uparrow \uparrow \uparrow \uparrow \uparrow \uparrow \uparrow \\
& 1,2,3,45,6, \ldots \frac{1}{2}-2, \frac{(1)}{2}-1, \frac{1}{2}
\end{aligned}
$$

concluding so (36) in a complete accordance with Postulate 3. Note that record (37) does not affirms that we have established the one-to-one correspondence among all even numbers and a half of natural ones. We cannot do this due to Postulate 1. The symbols '...' indicate an infinite number of numbers and we can execute only a finite number of operations. However, record (37) affirms that for any even number expressible in the chosen numeral system it is possible to indicate the corresponding natural number in the lower row of (37).

We conclude the paper by the following remark. With respect to our methodology, the mathematical results obtained by Pirahã, Cantor, and those presented in this paper do not contradict to each other. They all are correct with respect to mathematical languages used to express them. This relativity is very important and it has been emphasized in Postulate 2. For instance, the result of Pirahã $1+2=$ 'many' is correct in their language in the same way as the result $1+2=3$ is correct in the modern mathematical languages. Analogously, the result (36) is correct in Cantor's language and the more powerful language developed in this paper allows us to obtain a more precise result (37) that is correct in the new language.

The choice of the mathematical language depends on the practical problem that are to be solved and on the accuracy required for such a solution. Again, the result of Pirahã 'many' $+1=$ 'many' is correct. If one is satisfied with its accuracy, the answer 'many' can be used (and is used by Pirahã) in practice. However, if one needs a more precise result, it is necessary to introduce a more powerful mathematical language (a numeral system in this case) allowing one to express the required answer in a more accurate way.

\section{A Brief Conclusion}

In this paper, a new computational methodology has been introduced. It allows us to express, by a finite number of symbols, not only finite numbers but infinite and infinitesimals, too. All of them can be viewed as particular instances of a general framework used to express numbers.

It has been emphasized that the philosophical triad - researcher, object of investigation, and tools used to observe the object - existing in such natural sciences as Physics and Chemistry, exists in Mathematics, too. In natural sciences, the instrument used to 
observe the object influences the results of observations. The same happens in Mathematics where numeral systems used to express numbers are among the instruments of observations used by mathematicians. The usage of powerful numeral systems gives the possibility to obtain more precise results in Mathematics, in the same way as the usage of a good microscope gives the possibility to obtain more precise results in Physics.

\section{References}

Benci, V., and M. Di Nasso (2003). Numerosities of labeled sets: a new way of counting. Advances in Mathematics, 173, 50-67.

Cantor, G. (1955). Contributions to the Founding of the Theory of Transfinite Numbers. Dover Publications, New York.

Cohen, P.J. (1966). Set Theory and the Continuum Hypothesis. Benjamin, New York.

Conway, J.H., and R.K. Guy (1996). The Book of Numbers. Springer-Verlag, New York.

Gödel, K. (1931). Über formal unentscheidbare Sätze der Principia Mathematica und verwandter Systeme. Monatshefte für Mathematik und Physik, 38, 173-198.

Gödel, K. (1940). The Consistency of the Continuum-Hypothesis. Princeton University Press, Princeton.

Gordon, P. (2004). Numerical cognition without words: Evidence from Amazonia. Science, 306(15), October, 496-499.

Hardy, G.H. (1910). Orders of Infinity. Cambridge University Press, Cambridge.

Hilbert, D. (1902). Mathematical problems: Lecture delivered before the International Congress of Mathematicians at Paris in 1900. Bulletin of the American Mathematical Society, 8, 437-479.

Leibniz, G.W., and J.M. Child (2005). The Early Mathematical Manuscripts of Leibniz. Dover Publications, New York.

Markov Jr., A.A., and N.M. Nagorny (1996). Theory of Algorithms, 2 ed. FAZIS, Moscow.

Mockus, J. (2006). Investigation of examples of e-education environment for scientific collaboration and distance graduate studies: Part 1. Informatica, 17(1), 259-278.

Mockus, J. (2008). Investigation of examples of e-education environment for scientific collaboration and distance graduate studies: Part 2. Informatica, 19(1), 45-62.

Newton, I. (1671). Method of Fluxions.

Robinson, A. (1996). Non-Standard Analysis. Princeton Univ. Press, Princeton.

Sergeyev, Ya.D. (2003). Arithmetic of Infinity. Edizioni Orizzonti Meridionali, CS.

Sergeyev, Ya.D. (2004a). Computer system for storing infinite, infinitesimal, and finite quantities and executing arithmetical operations with them. Patent application 08.03.04, 2004.

Sergeyev, Ya.D. (2004b). http: / / www . theinfinitycomputer.com

Sergeyev, Ya.D. (2005). A few remarks on philosophical foundations of a new applied approach to Infinity. Scheria, 26-27, 63-72.

Sergeyev, Ya.D. (2006a). Mathematical foundations of the Infinity Computer. Annales UMCS Informatica AI, 4, 20-33.

Sergeyev, Ya.D. (2006b). Misuriamo l'infinito. Periodico di Matematiche, 6(2), 11-26.

Sergeyev, Ya.D. (2007). Blinking fractals and their quantitative analysis using infinite and infinitesimal numbers. Chaos, Solitons \& Fractals, 33(1), 50-75.

Strongin, R.G., and Ya.D. Sergeyev (2000). Global Optimization and Non-convex Constraints: Sequential and Parallel Algorithms. Kluwer Academic Publishers, Dordrecht.

Y.D. Sergeyev, $\mathrm{PhD}$, DSc, holds a full professorship reserved for distinguished scientists at the University of Calabria, Rende, Italy. He is also full professor (a part-time contract) at the N.I. Lobatchevsky State University, Nizhni Novgorod, Russia and affiliated researcher at the Institute of High Performance Computing and Networking of the National Research Council of Italy. 


\section{Naujas taikomasis būdas vykdyti skaičiavimus su begaliniais ir be galo mažais dydžiais}

Yaroslav D. SERGEYEV

Šiame straipsnyje aprašoma nauja skaičiuojamoji metodologija vykdyti skaičiavimus su begaliniais ir be galo mažais dydžiais. Ji yra pagrịsta senovès graiku pasiūlytu principu äDalis yra mažesnè už visumą" ir pritaikyta visiems skaičiams (baigtiniams, begaliniams ir be galo mažiems) bei visoms aibėms ir procesams (baigtiniams ir begaliniams). Yra parodyta, kad imanoma užrašyti baigtinius, begalinius ir be galo mažus skaičius baigtiniu kiekiu simbolių. Nauja metodologija igalino pasiūlyti begalybės kompiuterị, operuojantị tokiais skaičiais (jo imitatorius jau yra realizuotas). Pavyzdžiai su diverguojančiomis sekomis, begalinėmis aibèmis ir ribomis yra pateikti. 